contribute to the optimization of pharmacotherapy and to prioritising safety in an Intensive Care Unit (ICU).

Purpose To identify and quantify medicines errors observed and interventions made in the ICU in question, drawing a profile of the main actions of the pharmacist in critical care specialising in women's health.

Materials and Methods The study was conducted in a Brazilian ICU of a university hospital specialising in women's health, from February to May 2012. Interventions were performed after analysis of patient prescriptions (18 years old or over, hospitalised for more than 24 hours in the ICU) and discussions of clinical cases during multidisciplinary meetings. Interventions were classed on whether or not they were accepted by the medical staff. Drug-related errors observed were classed as preventable or not and ranked by an adaptation of the classification of the National Coordinating Council for Medication Error Reporting and Prevention (NCCMERP).

Results The study involved 82 patients, and 386 prescriptions were evaluated. The mean age was $41.1 \pm 19.0$ years old and the average hospital stay was $4.7 \pm 3.3$ days. We identified 45 medicines errors (mean $0.6 \pm 3.5$ /patient), $86.7 \%$ of these were preventable and $13.3 \%$ were not. The most common error types were: unsafe medicine due to drug interaction $(26.7 \%)$, higher dose than recommended (15.6\%) and unsafe medicine during lactation (13.3\%). Fifty-one interventions were made (mean $0.6 \pm 4.2 /$ patient), and $84.3 \%$ of these were accepted; $3.9 \%$ partially accepted; and $11.8 \%$ were not accepted. The most common interventions were to recommend an alternative dose (25.5\%), identify drug interactions (23.5\%), and risk during lactation (11.8\%).

Conclusions Partial results obtained show the necessity for clinical pharmacy services in the ICU as an important contribution to reducing risks from drug treatment.

No conflict of interest.

\section{CPC-109 PHARMACY INTRAVENOUS IRON PROTOCOL IN A CENTRAL HOSPITAL}

doi:10.1136/ejhpharm-2013-000276.566

1'J Amaral, 'A Parola, 'H Farinha, ${ }^{2} \mathrm{~F}$ Falcao. 'Hospital Egas Moniz-CHLO, Pharmacy, Lisbon, Portugal; ${ }^{2}$ Centro Hospitalar Lisboa Ocidental EPE, Pharmacy, Lisbon, Portugal

Background Iron deficiency anaemia (IDA) is a common condition. The pharmacy intravenous iron protocol $(100 \mathrm{mg} / 5 \mathrm{ml}$ iron sucrose vials) includes assessment of patient analytical data, dose calculation, schedule and information about iron administration intended to prevent adverse reactions.

Purpose To assess the use of intravenous iron in hospitalised patients being treated by the pharmacy protocol.

Materials and Methods An eight-month retrospective, observational study (January to August 2012). Hospitalized patients treated with pharmacist-managed intravenous iron were selected. Demography, main diagnosis, comorbidities, basic data, dosage suggestions and haemoglobin and haematocrit values were collected from electronic clinical files and pharmacotherapeutic profiles.

Results A total of 35 patients (19 male) were included. Mean age was 75.9 years (range 43-94).

$9(25.7 \%)$ patients were admitted for surgery and 26 (74.3\%) for a variety of medical conditions.

20 patients $(57.1 \%)$ were treated without complete investigation of the anaemia.

The most frequent intravenous iron dosage was $200 \mathrm{mg} 3 \mathrm{x}$ week.

$27(77.1 \%)$ patients had increased haemoglobin and haematocrit values after an average of 10.3 days (range 3-20) of intravenous iron replacement treatment. The mean increase in haemoglobin concentration was $2.5 \mathrm{~g} / \mathrm{dl}$ (range 0.2-6.6). Only 9 patients (25.7\%) achieved the haemoglobin target during admission. The majority of patients were discharge before achieving the target haemoglobin. No adverse reactions were reported to the pharmacist.

Conclusions As stated in the literature, a large proportion of patients in our study were not confirmed to be iron deficient. Pharmacist should advise physicians about the importance of a complete IDA study before starting this therapy. The information about iron administration and a test dose in the pharmacy protocol seem to be useful in preventing adverse reactions.

No conflict of interest.

\section{CPC-110 PHARMACY INVOLVEMENT IN THE MANAGEMENT OF ACADEMIC CLINICAL TRIALS}

doi:10.1136/ejhpharm-2013-000276.567

C Breuker, F Bringer, N Gastaut, M Naud, A Castet-Nicolas, S Hansel-Esteller. CHRU de Montpellier, Pharmacie Lapeyronie - Arnaud de Villeuneuve, Montpellier, France

Background The sponsor is the person or entity that initiates a clinical trial, manages it and provides funding. We define two types of promoters, commercial sponsors and academic sponsors (mainly hospitals). In order to minimise the cost of academic studies without limiting the quality, some work done by the hospital is not included, for example pharmaceutical management by pharmacies. Purpose To measure the size of pharmacy involvement in the management of clinical studies and academic costs not taken into account.

Materials and Methods We accounted for all pharmaceutical work done for academic studies (dispensing, preparation, reception of goods or materials, destruction of goods or materials, monitoring, labelling, ordering, randomization) managed by our pharmacy during the year 2011. We estimated the average time for each of these duties and the resulting financial cost (national grid, LEEM).

Results 35 institutional studies were in progress during this period and represented approximately $20 \%$ of all studies managed by our service: 8 studies were promoted by Montpellier hospitals, 7 by associations and 20 by other hospitals. We noted 501 prescriptions dispensed, 180 assignments to treatment or randomization, 52 preparations, 138 receptions, 13 destruction, 55 orders, 416 labels prepared and 52 monitoring visits. All this took 736.5 hours (or 210 half days) and additional costs estimated at 45,752 euros. Only 8,865 euros were allocated to the pharmacy (19\% of the costs).

Conclusions Academic research is essential and necessary for the improvement of scientific knowledge. However, in most cases, no expenditure is planned for the pharmacy unit. Currently, these activities are made within the hospital pharmacist's "free time". A national reflection is currently under way to establish a grid indicating how much academic studies should pay for the recruitment of dedicated medical staff. This study demonstrates that academic research requires a considerable time from the pharmacies, to justify the allocation of human resources in order to support good management.

No conflict of interest.

\section{CPC-111 PHARMACISTS' OPTIMIZATION OF THE MEDICATION PROCESS DURING ADMISSION TO HOSPITAL: A MULTICENTRE, RANDOMIZED, CONTROLLED TRIAL}

doi:10.1136/ejhpharm-2013-000276.568

'TRH Nielsen, ${ }^{2} \mathrm{PH}$ Honoré, ${ }^{3} \mathrm{SE}$ Andersen, ${ }^{4} \mathrm{M}$ Rasmussen. 'Region Zealand Hospital Pharmacy, Logistics and Clinical Pharmacy, Naestved, Denmark; ${ }^{2}$ School of Pharmaceutical Sciences, University of Copenhagen, Copenhagen, Denmark; ${ }^{3}$ Bispebjerg Hospital, Department of Clinical Pharmacology, Copenhagen, Denmark; ${ }^{4}$ No Affiliation - Freelance Author

Background During hospital admission, nearly $10 \%$ of all patients experience adverse events (AEs) and almost $1 / 3$ of AEs are 